\title{
The Cauchy-Kovalevskaya Extension Theorem in Discrete Clifford Analysis
}

\author{
H. De Ridder, H. De Schepper and F. Sommen \\ Clifford Research Group, Faculty of Engineering, Ghent University, Belgium
}

\begin{abstract}
Discrete Clifford analysis is a higher dimensional discrete function theory based on skew Weyl relations. It is centered around the study of Clifford algebra valued null solutions, called discrete monogenic functions, of a discrete Dirac operator, i.e. a first order, Clifford vector valued difference operator. In this contribution, we establish a Cauchy-Kovalevskaya extension theorem for discrete monogenic functions defined on the grid $\mathbb{Z}_{h}^{m}$ of $m$-tuples of integer multiples of a variable mesh width $h$. Convergence to the continuous case is investigated. As illustrative examples we explicitly construct the CauchyKovalevskaya extensions of the discrete delta function and of a discretized exponential.
\end{abstract}

Keywords: Cauchy-Kovalevskaya extension, discrete Clifford analysis

PACS: $02.30 . P x$

\section{INTRODUCTION}

The Cauchy-Kovalevskaya extension theorem $[1,5]$ is well-known; for a well-documented account on this result we refer to [2]. The idea behind the concept of Cauchy-Kovalevskaya extension (short: CK extension) is to characterize solutions of suitable (systems of) PDE's by their restriction, sometimes together with the restrictions of some of their derivatives, to a submanifold of codimension one.

In the complex plane, choosing the involved differential operator to be the Cauchy-Riemann operator, the CK extension theorem states that a holomorphic function in an appropriate region of the complex plane is completely determined by its restriction to the real axis. Moreover, given this restriction, the theorem also provides a reconstruction formula for the original function.

Euclidean Clifford analysis is a function theory which provides a higher dimensional generalization of the theory of holomorphic functions in the complex plane; the theory focusses on monogenic functions, i.e. Clifford algebra valued null solutions of the Dirac operator $\partial_{\underline{x}}=\sum_{k=1}^{m} e_{k} \partial_{x_{k}}$, where $\left(e_{1}, \ldots, e_{m}\right)$ is an orthonormal basis of $\mathbb{R}^{m}$, underlying the construction of the Clifford algebra. The CK extension theorem in this context thus is a direct generalization of the complex plane case; taking, in order to be in accordance with what follows, the signature of the Clifford algebra to be $(m, 0)$, it reads as follows.

Theorem 1 Identify $\mathbb{R}^{m-1}$ with $\left\{\underline{x} \in \mathbb{R}^{m}: x_{1}=0\right\}$, and let $\widetilde{f}\left(x_{2}, x_{3}, \ldots, x_{m}\right)$ be real analytic in an open set $\widetilde{\Omega}$ of $\mathbb{R}^{m-1}$, containing the origin. Then there exists an open neigbourhood $\Omega$ of $\widetilde{\Omega}$ in $\mathbb{R}^{m}$ and a unique monogenic function $f$ in $\Omega$, given by

$$
f\left(x_{1}, x_{2}, \ldots, x_{n}\right)=\exp \left(-x_{1} e_{1} \widetilde{\partial}_{\underline{x}}\right)[\widetilde{f}]=\sum_{k=0}^{\infty} \frac{1}{k !} x_{1}^{k}\left(-e_{1} \widetilde{\partial}_{\underline{x}}\right)^{k}[\widetilde{f}]
$$

such that its restriction to $\widetilde{\Omega}$ is precisely $\widetilde{f}$. Here $\widetilde{\partial}_{\underline{x}}$ stands for the restriction of $\partial_{\underline{x}}$ to $\mathbb{R}^{m-1}$.

In the paper [3] a discrete counterpart of Euclidean Clifford analysis, based on skew Weyl relations was constructed. Basic definitions were established for a discrete Dirac operator $D$, discrete monogenic functions and discrete spherical monogenics, i.e. homogeneous discrete monogenic polynomials. Now we establish a CK extension theorem in discrete Clifford analysis. The convergence of the discrete results to the corresponding continuous ones is investigated and illustrative examples are given. In an introductory section, see also [3], the basic framework for discrete Clifford analysis is included.

\footnotetext{
${ }^{1}$ The author acknowledges support by the institutional grant no. B/10675/02 of Ghent University (BOF).
} 


\section{BASIC NOTIONS OF THE DISCRETE SETTING}

Let $\mathbb{R}^{m}$ be the $m$-dimensional Euclidean space with orthonormal basis $\mathbf{e}_{j}, j=1, \ldots, m$. In the discrete Clifford setting, the equidistant lattice $\mathbb{Z}_{h}^{m}$ with general mesh width $h>0$ over the space $\mathbb{R}^{m}$ is introduced by $\mathbb{Z}_{h}^{m}=$ $\left\{\left(\ell_{1} h, \ell_{2} h, \ldots, \ell_{m} h\right) \mid\left(\ell_{1}, \ell_{2}, \ldots, \ell_{m}\right) \in \mathbb{Z}^{m}\right\}$. A Clifford vector $x$ thus will only be allowed to show co-ordinates which are integer multiples of the mesh width $h$. The one-sided forward and backward differences $\Delta_{j}^{ \pm}, j=1, \ldots, m$, act on a function $u$ as

$$
\Delta_{j}^{+}[u]=\frac{u\left(\cdot+h \mathbf{e}_{j}\right)-u(\cdot)}{h}, \quad \Delta_{j}^{-}[u]=\frac{u(\cdot)-u\left(\cdot-h \mathbf{e}_{j}\right)}{h}
$$

and are seen as 'lowering operators'. The corresponding discrete (star) Laplacian is given by

$$
\Delta_{h}^{*}[f](\underline{x})=\sum_{j=1}^{m} \Delta_{j}^{+} \Delta_{j}^{-}[u]=\sum_{j=1}^{m}\left(\frac{f\left(\underline{x}+h \mathbf{e}_{j}\right)-f\left(\underline{x}-h \mathbf{e}_{j}\right)}{h^{2}}\right)-2 m \frac{f(\underline{x})}{h^{2}}
$$

To introduce the discrete Dirac operator, the so-called Hermitean setting is used, where the Clifford algebra $\mathbb{R}_{0, m}$ is embedded in a bigger complex Clifford algebra $\mathbb{C}_{2 m}$, the underlying vector space of which has double the dimension. This allows us to split each basis element $\mathbf{e}_{j}$ into a forward and backward basis element $\mathbf{e}_{j}^{+}$and $\mathbf{e}_{j}^{-}$, satisfying the anti-commutator relations $\mathbf{e}_{j}^{-} \mathbf{e}_{\ell}^{-}+\mathbf{e}_{\ell}^{-} \mathbf{e}_{j}^{-}=0, \mathbf{e}_{j}^{+} \mathbf{e}_{\ell}^{+}+\mathbf{e}_{\ell}^{+} \mathbf{e}_{j}^{+}=0$ and $\mathbf{e}_{j}^{+} \mathbf{e}_{\ell}^{-}+\mathbf{e}_{\ell}^{-} \mathbf{e}_{j}^{+}=\delta_{j \ell}$. The discrete Dirac operator

$$
D_{h}[f](\underline{x})=\sum_{j=1}^{m}\left(\mathbf{e}_{j}^{+} \frac{f\left(\underline{x}+h \mathbf{e}_{j}\right)-f(\underline{x})}{h}+\mathbf{e}_{j}^{-} \frac{f(\underline{x})-f\left(\underline{x}-h \mathbf{e}_{j}\right)}{h}\right)
$$

factorizes the star Laplacian $\left(D_{h}^{2}=\Delta_{h}^{*}\right)$ and tends to $\partial_{\underline{x}}$ as $h \rightarrow 0$. A discrete functions then is called discrete monogenic iff it is in the kernel of the discrete Dirac operator. 'Raising operators' $X_{j}^{ \pm}$, interacting with the corresponding 'lowering operators' $\Delta_{j}^{ \pm}$according to the skew Weyl relations $\Delta_{j}^{+} X_{j}^{+}-X_{j}^{-} \Delta_{j}^{-}=1$ and $\Delta_{j}^{-} X_{j}^{-}-X_{j}^{+} \Delta_{j}^{+}=1$, combine into the discrete vector variable $X=\sum_{j=1}^{m}\left(\mathbf{e}_{j}^{+} X_{j}^{-}+\mathbf{e}_{j}^{-} X_{j}^{+}\right)$. The discrete Euler operator $E$, see also [3], has the explicit form $E=\sum_{j=1}^{m}\left(\mathbf{e}_{j}^{+} \mathbf{e}_{j}^{-} X_{j}^{-} \Delta_{j}^{-}+\mathbf{e}_{j}^{-} \mathbf{e}_{j}^{+} X_{j}^{+} \Delta_{j}^{+}\right)$and satisfies intertwining relations with the Dirac operator and the vector variable which also hold in the continuous Clifford setting, i.e. $D X+X D=2 E+m, D E=E D+D$ and $E X=X E+X$.

Discrete homogeneous polynomials of degree $k$, also called discrete spherical monogenics, are, by definition, the eigenfunctions of the discrete Euler operator with eigenvalue $k$. In order to determine them, we decompose $X$ into coordinate variables $\xi_{j}=\mathbf{e}_{j}^{+} X_{j}^{-}+\mathbf{e}_{j}^{-} X_{j}^{+}$, and $D$ into co-ordinate difference operators $\partial_{j}=\mathbf{e}_{j}^{+} \Delta_{j}^{+}+\mathbf{e}_{j}^{-} \Delta_{j}^{-}$, which inherit their (anti)commutator relations from the skew Weyl relations, namely $\partial_{j} \xi_{j}-\xi_{j} \partial_{j}=1$ and $\partial_{\ell} \xi_{j}+\xi_{j} \partial_{\ell}=0, \ell \neq j$. Invoking the intertwining relation between $E$ and $X$, it is easily seen that $E \xi_{j}=\xi_{j}(E+1)$, whence the natural powers of the operator $\xi_{j}$ acting on the ground state $1, \xi_{j}^{k}[1]$, are the basic discrete homogeneous polynomials of degree $k$ in the variable $x_{j}$, similar to $x_{j}^{k}$ in the continuous setting. The homogeneous discrete polynomials $\xi_{j}^{k}[1]$ are explicitly given by $\xi_{j}[1]\left(x_{j}\right)=x_{j}\left(\mathbf{e}_{j}^{+}+\mathbf{e}_{j}^{-}\right)$and, for $n=1,2, \ldots$ and $j=1, \ldots, m$,

$$
\xi_{j}^{2 n+1}[1]\left(x_{j}\right)=x_{j}\left(\mathbf{e}_{j}^{+}+\mathbf{e}_{j}^{-}\right) \prod_{i=1}^{n}\left(x_{j}^{2}-h^{2} i^{2}\right), \quad \xi_{j}^{2 n}[1]\left(x_{j}\right)=\left(x_{j}^{2}+n h x_{j}\left(\mathbf{e}_{j}^{+} \mathbf{e}_{j}^{-}-\mathbf{e}_{j}^{-} \mathbf{e}_{j}^{+}\right)\right) \prod_{i=1}^{n-1}\left(x_{j}^{2}-h^{2} i^{2}\right)
$$

\section{THE DISCRETE CAUCHY-KOVALEVSKAYA EXTENSION}

Let $f$ be a discrete function in the variables $\left(x_{2}, \ldots, x_{m}\right)$, defined on the grid $\mathbb{Z}_{h}^{m-1}$ and taking values in the algebra over $\left\{\mathbf{e}_{2}^{+}, \mathbf{e}_{2}^{-}, \ldots, \mathbf{e}_{m}^{+}, \mathbf{e}_{m}^{-}\right\}$. Then it can be shown that there exists a unique discrete monogenic function, called the Cauchy-Kovalevskaya extension (short CK extension) of $f$, in the variables $\left(x_{1}, \ldots, x_{m}\right)$, defined on the grid $\mathbb{Z}_{h}^{m}$ and taking values in the algebra over $\left\{\mathbf{e}_{1}^{+}, \mathbf{e}_{1}^{-}, \ldots, \mathbf{e}_{m}^{+}, \mathbf{e}_{m}^{-}\right\}$, such that its restriction to $x_{1}=0$ equals $f$. Explicitely, this CK extension is given by

$$
\mathrm{CK}[f]\left(x_{1}, x_{2}, \ldots, x_{m}\right)=\sum_{k=0}^{\infty} \frac{\xi_{1}^{k}[1]\left(x_{1}\right)}{k !} f_{k}\left(x_{2}, \ldots, x_{m}\right)
$$

where, denoting the restriction of $D_{h}$ to $\mathbb{Z}_{h}^{m-1}$ by $D_{h}^{\prime}$, it holds that $f_{0}=f$ and $f_{k+1}=(-1)^{k+1} D_{h}^{\prime} f_{k}$. 
Note that, in this definition there are no conditions imposed on the original function $f$, since from (1) it follows that $\xi_{1}^{2 n+1}[1]\left(x_{1}\right)=0$, for $n \geqslant \frac{\left|x_{1}\right|}{h}$ and $\xi_{1}^{2 n}[1]\left(x_{1}\right)=0$ for $n \geqslant \frac{\left|x_{1}\right|}{h}+1$, implying that for every point $\left(x_{1}, \ldots, x_{m}\right)$ of the grid $\mathbb{Z}_{h}^{m}$, the series reduces to a finite sum.

As $h$ approaches zero, $D_{h}$ tends to $\partial_{x}$ while $\xi_{j}^{2 n+1}[1]\left(x_{1}\right) \rightarrow x_{j}^{2 n+1} e_{j}$ and $\xi_{j}^{2 n}[1]\left(x_{1}\right) \rightarrow x_{j}^{2 n}$. Formally, the discrete CK extension thus tends to the corresponding continuous CK extension. However, convergence depends on the considered function, since the continuous CK extension only exists for real analytic functions. For example, the CK extension of a delta function exists only in the discrete setting; for $h \rightarrow 0$ the values will diverge. On the other hand, the discrete counterpart of the real analytic function $\exp \left(x_{2}\right)$ admits a CK extension that converges to the continuous one when $h$ approaches zero. Both cases are illustrated below.

\section{ILLUSTRATIVE EXAMPLES}

\section{CK extension of the discrete delta function}

As a first example, we present the $\mathrm{CK}$ extension of the discrete delta function, i.e. the restriction to the grid $\mathbb{Z}_{h}^{m-1}$ of the function $\delta_{0}\left(x_{2}, \ldots, x_{m}\right)$ on $\mathbb{R}^{m-1}$, taking the value 1 in $(0, \ldots, 0)$ and the value 0 elsewhere. As every discrete function given by its values in the vertices of the grid can be written as a linear combination of shifted delta functions, the CK extension of the delta function is a basic building block for the CK extension of other functions. We will only treat the case $m=2$ explicitely; however, other dimensions may be directly computed as well.

The CK extension of $\delta_{0}\left(x_{2}\right)$ is given by $C K\left[\delta_{0}\right]\left(x_{1}, x_{2}\right)=\sum_{k=0}^{\infty} \frac{\xi_{1}^{k}[1]\left(x_{1}\right)}{k !} f_{k}\left(x_{2}\right)$ where $f_{0}\left(x_{2}\right)=\delta_{0}\left(x_{2}\right)$, and

$$
\begin{aligned}
& f_{2 n}\left(x_{2}\right)=\sum_{j=0}^{2 n}(-1)^{j+n}\left(\begin{array}{c}
2 n \\
j
\end{array}\right) \frac{\delta_{(n-j) h}}{h^{2 n}} \\
& f_{2 n+1}\left(x_{2}\right)=\mathbf{e}_{2}^{+}\left(\sum_{j=0}^{2 n+1}(-1)^{j+n+1}\left(\begin{array}{c}
2 n+1 \\
j
\end{array}\right) \frac{\delta_{(j-n-1) h}}{h^{2 n+1}}\right)+\mathbf{e}_{2}^{-}\left(\sum_{j=0}^{2 n+1}(-1)^{j+n}\left(\begin{array}{c}
2 n+1 \\
j
\end{array}\right) \frac{\delta_{(n+1-j) h}}{h^{2 n+1}}\right)
\end{aligned}
$$

This allows to calculate the scalar part of $\operatorname{CK}\left[\delta_{0}\right]$, which is depicted in figure 1 . For a point $\left(x_{1}, x_{2}\right)$ of the grid, it is given by

$$
1+\sum_{n=1}^{\left|\frac{x_{1}}{h}\right|} \frac{x_{1}^{2} \prod_{m=1}^{n-1}\left(x_{1}^{2}-m^{2} h^{2}\right)}{h^{2 n}(n !)^{2}} \text { when } x_{2}=0, \quad(-1)^{\left|\frac{x_{2}}{h}\right|} \sum_{n=\left|\frac{x_{2}}{h}\right|}^{\left|\frac{x_{1}}{h}\right|} \frac{x_{1}^{2} \prod_{m=1}^{n-1}\left(x_{1}^{2}-m^{2} h^{2}\right)}{h^{2 n}\left(n-\frac{x_{2}}{h}\right) !\left(n+\frac{x_{2}}{h}\right) !} \text { when } x_{2} \neq 0
$$

When $h$ approaches zero, the values of $\mathrm{CK}\left[\delta_{0}\left(x_{2}\right)\right]\left(x_{1}, x_{2}\right)$ increase in absolute value to infinity, for every point of the grid $\left(x_{1}, x_{2}\right)$ with $x_{1} \neq x_{2}$. Note that the continuous function $\delta_{0}$ is not real analytic and hence has no CK extension in the continuous setting.
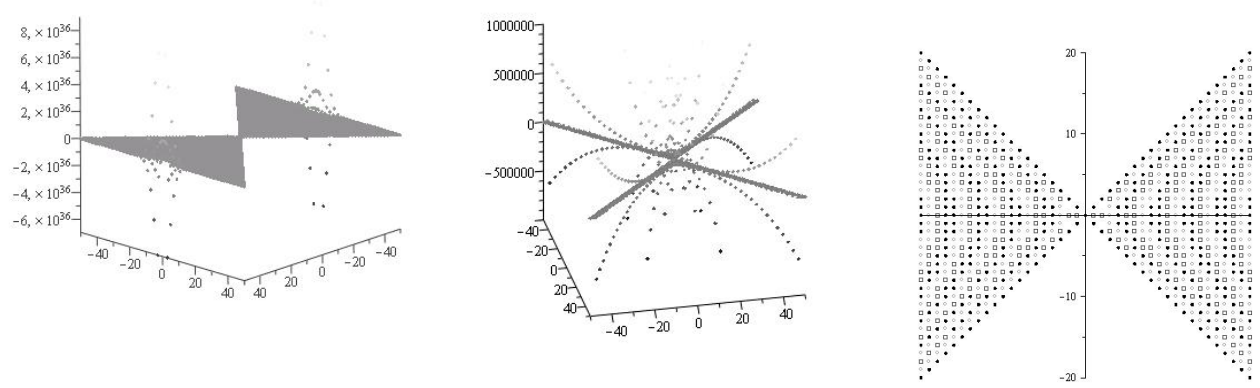

FIGURE 1. Scal $\left(\mathrm{CK}\left[\delta_{0}\right]\left(x_{1}, x_{2}\right)\right)$, first fully displayed, then zoomed in and finally given by a contourplot 


\section{CK extension of a discrete exponential}

Again we restrict ourselves to dimension $m=2$. The CK extension of the restriction of the real analytic function $\exp \left(x_{2}\right)$ to $\mathbb{Z}_{h}$, is given by

$$
\mathrm{CK}_{h}\left[\exp \left(x_{2}\right)\right]\left(x_{1}, x_{2}\right)=\exp \left(x_{2}\right)+\sum_{k=1}^{\left|\frac{x_{1}}{h}\right|} \frac{\left(\xi_{1}\right)_{h}^{2 k}[1]\left(x_{1}\right)}{(2 k) !} f_{2 k}\left(x_{2}\right)+\sum_{k=0}^{\left|\frac{x_{1}}{h}-1\right|} \frac{\left(\xi_{1}\right)_{h}^{2 k+1}[1]\left(x_{1}\right)}{(2 k+1) !} f_{2 k+1}\left(x_{1}\right)
$$

where the functions $f_{k}$ are given by $f_{0}=\exp \left(x_{2}\right), f_{2 k}=\frac{(-1)^{k}}{h^{2 k}} \sum_{j=0}^{2 k}(-1)^{j}\left(\begin{array}{c}2 k \\ j\end{array}\right) \exp \left(x_{2}+(k-j) h\right)$ and

$$
f_{2 k+1}=\frac{(-1)^{k+1}}{h^{2 k+1}}\left[\mathbf{e}_{2}^{+} \sum_{j=0}^{2 k+1}(-1)^{j}\left(\begin{array}{c}
2 k+1 \\
j
\end{array}\right) \exp \left(x_{2}+(k+1-j) h\right)-\mathbf{e}_{2}^{-} \sum_{j=0}^{2 k+1}(-1)^{j}\left(\begin{array}{c}
2 k+1 \\
j
\end{array}\right) \exp \left(x_{2}-(k+1-j) h\right)\right]
$$

For a point $\left(x_{1}, x_{2}\right)$ of the grid $\mathbb{Z}_{h}^{2}$, the scalar part of $\mathrm{CK}_{h}\left[\exp \left(x_{2}\right)\right]\left(x_{1}, x_{2}\right)$ is given by

$$
\exp \left(x_{2}\right)\left[1+\sum_{k=1}^{\left|\frac{x_{1}}{h}\right|}(-1)^{k}\left(\frac{x_{1}^{2} \prod_{m=1}^{k-1}\left(x_{1}^{2}-(m h)^{2}\right)}{(2 k) ! h^{2 k}} \sum_{m=-k}^{k}\left((-1)^{m}\left(\begin{array}{c}
2 k \\
k-m
\end{array}\right) \exp (m h)\right)\right]\right.
$$

When $h$ approaches zero, the CK extension of the discrete function very quickly approaches the CK extension of the continuous function. Figure 2 depicts the values of both the continuous as the discrete CK extension of $\exp (x 2)$, for two different values of $h$.

\section{REFERENCES}

1. A. Cauchy, Oeuvres completes, série 1, Tome VII, Gauthier-Villars (Paris, 1882-1974), 17-58.

2. R. Cooke, The Cauchy-Kovalevskaya Theorem, preprint, available online: http://www.cems.uvm.edu/ cooke/ckthm.pdf.

3. H. De Ridder, H. De Schepper, U. Kähler, F. Sommen, Discrete function theory based on skew Weyl relations, Proceedings of AMS 138 (2010), 3241-3256.

4. N. Faustino, U. Kähler, F. Sommen, Discrete Dirac operators in Clifford analysis, Adv. Appl. Cliff. Alg. 17(3) (2007), $451-467$.

5. S. Kowalevsky, Zur Theorie der partiellen Differentialgleichung, J. für die Reine und Angew. Mathem. 80 (1875), 1 Ú-32.
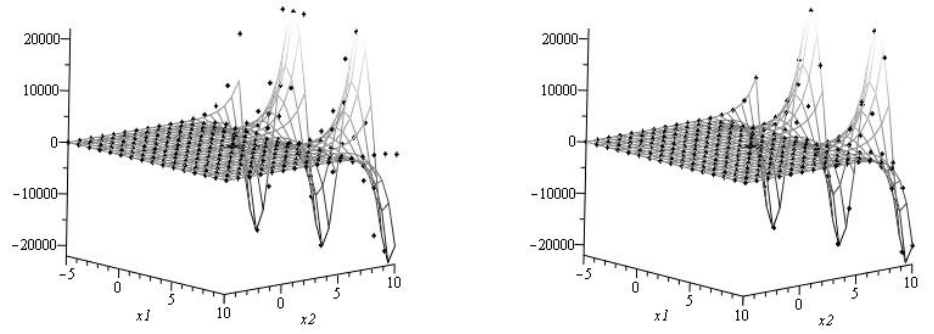

FIGURE 2. Scal $\left(\mathrm{CK}\left[\exp \left(x_{2}\right)\right]\left(x_{1}, x_{2}\right)\right)$ both discrete and continuous, for $h=1$ and $h=\frac{1}{10}$ 